\title{
The Distribution of Work-Related Musculoskeletal Disorders Among Nurses in Sub-Saharan Africa: A Scoping Review Protocol
}

Kagiso Kgakge ( $\nabla$ kagisoh@yahoo.com )

Gaborone Institute of Health Sciences https://orcid.org/0000-0001-8858-3906

\section{Mbuzeleni Hlongwa}

University of KwaZulu-Natal School of Clinical Medicine

Themba Ginindza

University of KwaZulu-Natal School of Nursing and Public Health

\section{Protocol}

Keywords: Musculoskeletal disorders, nurses, Low back ache, hospitals, sub Saharan Africa

Posted Date: February 10th, 2021

DOI: https://doi.org/10.21203/rs.3.rs-50166/v2

License: (c) (i) This work is licensed under a Creative Commons Attribution 4.0 International License. Read Full License

Version of Record: A version of this preprint was published at Systematic Reviews on August 13th, 2021. See the published version at https://doi.org/10.1186/s13643-021-01774-7. 


\section{Abstract}

\section{Background}

Although measures have been put in place, musculoskeletal injuries are noticeable high among the nursing fraternity with Low back pain (LBP) being the most prevalent. It is evident that healthcare professionals are in constant exposure to occupational hazards such as musculoskeletal injuries as they discharge their professional duties. Not only does LBP affect the health of the nurses, it also creates a huge burden on the health systems with consequent poor performance at the workplace as well as economic burden. Therefore, the main objective of this study is to map evidence on the prevalence, incidence, mortality, risk factors and economic costs of musculoskeletal disorders (MSD) in sub Saharan Africa (SSA).

\section{Methods and analysis}

Scoping review will be done to explore, describe and map literature on the prevalence, incidence, mortality, risk factors, and economic costs related to MSD among nurses in SSA. The search will be done using databases such as PubMed, MEDLINE, CINAHL, Google Scholar, nursing academic editions and World Health Organization (WHO) library databases. The search will look for primary studies within peer-reviewed articles as well as gray literature. In addition, the researcher will search the articles using keywords from the included studies as well as the list of references for related studies. The screening will be guided by the inclusion and exclusion criteria and the scoping review will be reported in accordance to PRISMA-P guidelines. The NVivo 12 data analysis software will be used to generate themes, and a thematic content analysis will be used to give the narrative account of the review.

\section{Discussion}

At the end, this study anticipates to uncover the relevant literature in SSA in regard to prevalence, incidence, mortality, risk factors and costs related to MSD among nurses; furthermore findings from this study will help in identifying research gaps, informing policy, priority in funding and planning and to guide future research.

\section{Background}

Work- related musculoskeletal disorders (WMSD) are described as musculoskeletal disorders that arise from work related events(1). On the other hand, Musculoskeletal disorders (MSD) is defined as a condition that include a gamut of inflammatory and degenerative conditions that affects the tendons, muscles, joints, ligaments, peripheral nerves, and supporting blood vessels with consequent pain, ache or discomfort (2). Literature indicates that MSD have increasingly become a major public health problem and a significant leading occupational burden among the nurses globally.

Epidemiological studies revealed that MSD are a major occupational health problem in the nursing fraternity due to the nature of its job (3). A number of factors have been related to the development of MSD among nurses; Physical factors like manual handling of patients and long-standing hours; Psychosocial factors like stress, anxiety and depression; Organizational factors like working shifts, shortage of staff and poor working conditions $(4,5)$. Therefore, MSD pose a major threat to the quality of life for nurses, resulting in work absenteeism, work limitations and ultimate need to change jobs

(6). Research findings reveal a noticeable high prevalence of LBP being the most reported MSD among nurses ranging from $33 \%$ to $90.1 \%$ globally. Although LBP is regarded as an insignificant condition especially in SSA, the Global Burden of Disease (GBD 2015) has indicated that LBP is the leading cause of disability associated with a significant amount of cost. Furthermore, limited research around this field has prevailed with more attention focusing on epidemic infectious diseases such as HIV/AIDS, TB, and Malaria in the African region(7).

Despite numerous efforts and measures that have been put in place to help curb the prevalence of MSD among nurses, the prevalence of MSD still prevails. It is therefore possible that the findings to this study will address this knowledge gap with documented evidence on the burden of MSD among nurses in SSA as well as advocate for sound strategies to tackle the

Page $2 / 9$ 
disorder like governments coming up with ergonomic interventions such as zero lifting policy, exercise programs and regular assessment of workplaces at regular intervals by safety and health professionals to ensure that all necessary preventative measures are implemented to alleviate the impact of the disease to the nurses.

Therefore, this scoping review will seek to find the evidence on prevalence, incidence, mortality, associated risk factors and economic costs on MSD among nurses with the aim of developing interventions directed at controlling and preventing the development of MSD among nurses in SSA, with further emphasis on change of the research focus and funding directions in this field.

\section{Methods And Materials}

The proposed scoping review will be conducted according to Preferred Reporting Items for Systematic Reviews and MetaAnalysis Protocols (PRISMA-P) guidelines(8).

Scoping review frameworkThe proposed framework by Arksey H \& O'Malley will be adopted for this scoping review(9). The framework involves (I) identifying the research question, (II) identifying relevant studies, (III) study selection, (IV) charting the data, and (V) collating, summarizing, and reporting the results

\section{Identifying the research question}

The main research question is: "What is the evidence on the burden of work-related musculoskeletal disorders in SubSaharan Africa?

\section{Research sub-questions will be as follows:}

- What is the burden of WMSDs among nurses in Sub-Saharan Africa with estimation in relation to prevalence, incidence and mortality?

- What are the risk factors associated with WMSD?

- What are the economic costs associated with WMSD?

\section{Eligibility criteria}

Studies will be selected according to the PEO (population, exposure, and outcomes) framework outlined below (Table 1)

Table 1: PEO framework for eligibility of research question

\begin{tabular}{ll}
\hline Criteria & Determinants \\
\hline Population and their problem & Nurses with MSD in Sub Saharan Africa \\
Exposure & Hospital work \\
Outcome & 1.Prevalence \\
& 2.Incidence \\
& 3.Mortality \\
& 4.Risk factors \\
& 5.Economic costs \\
& 6.Low back ache \\
\hline
\end{tabular}

\section{Search strategy}


Keyword search will be conducted from various electronic databases without a date limit; PubMed, CINAHL, Google Scholar, nursing academic editions and World Health Organization (WHO) library databases. Furthermore, we will work closely with the University of KwaZulu-Natal library services during the database searching and retrieval to improve the quality of our search strategy of articles. Keywords that will be used to search for these databases are as follows: work related musculoskeletal disorders, nurses, musculoskeletal disorders, low back ache, prevalence, incidence, mortality, economic costs related to MSD and risk factors. During the search, keywords will be separated by Boolean terms (AND, OR). MESH terms will also be used. Furthermore, the reference lists of studies eligible for inclusion will be screened for potential additional articles. Due to under-representation of literature in this area, we will do a global search approach then contextual interpretation for Sub-Saharan Africa. Sub-Saharan African country names and truncated terms such as west Africa, east Africa or southern Africa will be used to retrieve articles indexed using African country specific names or regional terms are retrieved (Appendix I).

\section{Study selection}

Two independent reviewers will conduct screening, guided by the eligibility criteria for this review. The support from the University of KwaZulu-Natal library services will be sought during database searching and retrieval of articles. The PRISMA (preferred reporting items for systematic reviews and meta-analysis) flow diagram will be used to document the review process (8). Articles with relevant title to the subject of the research will be uploaded to Endnote version X7 X7.7.1. The titles

and abstracts that do not meet the study eligibility criteria will be excluded. Discrepancies between reviewers will be resolved through consensus and will involve a third reviewer.

\section{Eligibility criteria}

\section{Inclusion criteria}

- Study will include nurses with low back ache

- All the study designs with relevant intervention

- Studies that focus on work-related musculoskeletal disorders among nurses; prevalence, incidence, mortality, economic costs and risk factors.

- Only studies conducted in English and in other languages with an English version will be included.

\section{Exclusion criteria}

- Studies that do not focus on nurses and low back ache

- Studies that do not focus on work-related musculoskeletal disorders among nurses

- Studies conducted outside Sub-Saharan Africa

- Studies published in languages other than English and do not have an English version will also be excluded.

\section{Study limitation}

We have got limitations as far as the language is concerned because we use English in our setting as it is the language that we understand, hence the potential to exclude relevant articles that are published in other languages.

PRISMA flow chart will be used to report the screening results

Table 2 will be used to show the results of the titles searched from different databases. 
\begin{tabular}{|l|l|l|l|l|}
\hline Date of search & Search engine & Keyword search & Number of articles found & Number articles eligible \\
\hline
\end{tabular}

PRISMA flow chart (Additional file 1: Figure S1) will be used to summarize the inclusion and exclusion criteria.

\section{Charting the data}

The relevant data will be drawn out using an extraction form (Appendix 2). In addition to answering the research question a charting form will be developed in such a way that it covers all the needed variables and it will be regularly updated when need arise. The extraction form will continually be updated. Then the form (Table 3) which includes the following: author with date, study title, study design, study setting, population, study aim, intervention, percentages, outcomes of the study, key findings, and comments will be used to chart the data of all the reviewed articles, coded using a coding system. This is purposively done to keep track of the studies included and excluded during the charting process of the scoping review.

Table 3: Data charting form

\begin{tabular}{l}
\hline Author and date \\
\hline $\begin{array}{l}\text { Study design } \\
\text { Study setting } \\
\text { Population }\end{array}$ \\
- Average age \\
- Sample size \\
Aims \\
Intervention \\
Outcome \\
Key findings \\
Conclusions \\
Comment \\
\hline
\end{tabular}

\section{Collating, summarizing, and reporting the results.}

The proposed scoping review aims to map the existing evidence on the distribution of work-related musculoskeletal disorders among nurses in Sub-Saharan Africa and to sum up the findings as presented in the relevant articles. All data relating to prevalence of MSD and low back ache, incidence of MSD, mortality of MSD, risk factors of MSD, economic costs related to MSD will be extracted and structured for the purpose of identifying themes. A descriptive report of the findings from literature will be presented through thematic content analysis. The structure of the literature will assume the following estimates; prevalence, incidence, mortality, economic cost and risk factors. The explanation of the findings will be analyzed to see how they relate to the overall study purpose and the implications of these findings for future research, policy, and practice.

\section{Quality assessment}

Assessment of the evidence from different studies will be conducted for quality, this will be done in order to ensure appropriateness of the study design to the research objectives and to minimize risk of bias. A quality appraisal tool which focuses on the study methods, the Mixed Method Quality Appraisal Tool (MMAT) Version 2011, will be used (10) . The tool will be used to examine the quality of an article looking at the following aspects: the appropriateness of the aim of the study, 
adequacy and methodology, study design, participant recruitment, data collection, data analysis, presentation of findings, authors' discussions, and conclusions.

\section{Discussion}

Work related musculoskeletal disorders (WMSD) has emerged to be a major public health concern globally, therefore the proposed scoping review seek to map existing evidence regarding the distribution (prevalence, incidence and mortality), risk factors and estimated costs associated to MSD among nurses in SSA in order to publish the research gaps in this area which has sparse data. According to (2), MSD are the most common cause of morbidity among the healthcare workers especially the nurses. The high prevalence of MSD among nurses has been linked to the physical nature of their job and the organizational factors by many researchers $(11,12)$. The burden of MSD among nurses is commonly associated with a number of factors such as lifting patients, lifting heavy loads, patient transfer from the floor and out of bed and working in awkward postures resulting in acute and accumulative MSD.

This study will map the evidence on the distribution of MSD with emphasis on the trends of low back pain (LBP) among nurses. Most studies that have been conducted on MSD among nurses indicate that LBP is the most prevalent and a major cause of disability affecting the general well-being and performance. The 2010 Global Burden of Disease Study estimated that LBP is among the top 10 diseases and injuries that account for the highest number of Disability Adjusted Life Years (DALY) worldwide resulting in activity limitation and work absence with consequent high economic burden on individuals, families, communities, industry, and governments (13).

It is critical to map the evidence of MSD among nurses as this will bring out more understanding towards this disease with the aim of directing more efforts towards the health of healthcare workers, policy makers and all relevant stakeholders to fight this scourge. Furthermore, this review will uproot an economic projection of LBP and a coherent healthcare resource allocation as well as intervention program towards MSD in order to minimize the severity and impact on healthcare workers.

\section{Conclusion}

Findings from this proposed scoping review are expected to give a reflection on the distribution MSD among nurses in the SSA region with estimates on prevalence, incidence, mortality with the identification of risk factors, and the associated economic burden. Therefore, the evidence synthesized from this study will help researchers, decision makers, and other stakeholders to inform policy and ensure an efficient allocation of healthcare resources, improving the healthcare system performance and thus improving treatment and reduce mortality associated.

\section{Abbreviations}

DALY: Disability Adjusted Life Year

GBD: Global burden of disease

LBP: Low back pain

MMAT: Mixed Method Quality Appraisal Tool

MSD: Musculoskeletal disorder

PRISMA-P: Preferred Reporting Items for Systematic Reviews and Meta-Analysis Protocols

SSA: Sub-Saharan Africa

WHO: World Health Organization 
WMSD: Work related musculoskeletal disorders

\section{Declarations}

Acknowledgements:

The authors would like to thank the University of KwaZulu-Natal (UKZN) for the provision of resources towards this review and the UKZN Systematic Review Unit for the training and technical support.

\section{Funding:}

Not applicable

\section{Availability of data and materials:}

All data generated or analyzed during this study will be included in the published systematic review article.

\section{Contributions:}

KK conceptualized the study and designed data collection methods under the supervision of TG. KK, TG and MH contributed to writing the first draft of the manuscript. All authors critically reviewed and approved of the final manuscript.

\section{Ethics approval and consent to participate:}

Not applicable

\section{Consent for publication:}

Not applicable

\section{Competing interests:}

The authors declare that they have no competing interests.

\section{References}

1. Salik Y, Özcan A. Work-related musculoskeletal disorders: A survey of physical therapists in Izmir-Turkey. BMC Musculoskelet Disord. 2004;5:1-7.

2. Punnett L, Wegman DH. Work-related musculoskeletal disorders: The epidemiologic evidence and the debate. J Electromyogr Kinesiol. 2004;14(1):13-23.

3. Burdorf A, Sorock G. Positive and negative evidence of risk factors for back disorders. Scand J Work Environ Heal. 1997;23(4):243-56.

4. J Smedley, H Inskip, F Trevelyan, P Buckle, C Cooper DC. nurses. 2003;864-9.

5. Rathore FA, Attique R, Asmaa Y. Prevalence and Perceptions of Musculoskeletal Disorders Among Hospital Nurses in Pakistan: A Cross-sectional Survey. Cureus. 2017;9(1):1-10.

6. Choi SD, Brings K. Work-related musculoskeletal risks associated with nurses and nursing assistants handling overweight and obese patients: A literature review. Work. 2016;53(2):439-48.

7. Kahere M, Ginindza T. Mapping evidence on the prevalence, incidence, risk factors and cost associated with chronic low back pain among adults in Sub-Saharan Africa: A systematic scoping review protocol. Syst Rev. 2020;9(1):1-9.

8. AC T, Lillie E Z, W OK, H C, D L, et al. Supplementary Table S3: PRISMA-ScR Checklist . Ann Intern Med [Internet]. 2018;169(7):11-2. Available from: http://www.prisma-statement.org/Extensions/ScopingReviews 
9. Arksey H, O’Malley L. Scoping studies: Towards a methodological framework. Int J Soc Res Methodol Theory Pract. 2005;8(1):19-32.

10. Pluye P, Robert E, Cargo M, Bartlett G. Proposal: A mixed methods appraisal tool for systematic mixed studies reviews. Montréal McGill Univ [Internet]. 2011;(Part I):1-8. Available from:

http://mixedmethodsappraisaltoolpublic.pbworks.com/f/MMAT 2011 criteria and tutorial 2011-06-

29.pdf\%5Cnhttp://scholar.google.com/scholar?

hl=en\&btnG=Search\&q=intitle:Proposal:+A+mixed+methods+appraisal+tool+for+systematic+mixed+studies+reviews. \#0

11. Tinubu BM, Mbada CE, Oyeyemi AL, Fabunmi AA. Work-related musculoskeletal disorders among nurses in Ibadan, South-west Nigeria: A cross-sectional survey. BMC Musculoskelet Disord. 2010;11:6-13.

12. Mbada CE, Obembe AO, Alade BS, Adedoyin RA, Awotidebe TO, Johnson OE, et al. Nijerya'da Bir Eğitim Hastanesinde Sağlık Çalışanları Arasında İş ile İlişkili Kas İskelet Bozuklukları [Work-Related Musculoskeletal Disorders among Health Workers in a Nigerian Teaching Hospital. TAF Prev Med Bull Res Artic TAF Prev Med Bull [Internet]. 2012;11(115):583-8. Available from: https://www.ejmanager.com/mnstemps/1/1-1320331223.pdf?t=1526599286

13. Reinhart L, Knight W, Roberts L, Mendes C. HHS Public Access Global Burden of Disease Study 2010. Lancet. 2012;380(9859):2163-96.

\section{Figures}

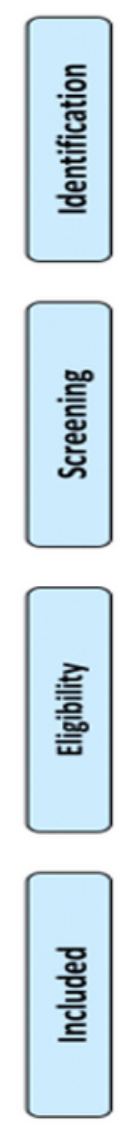

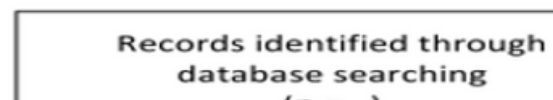
$(n=)$
Additional records identified

through other sources $(n=)$
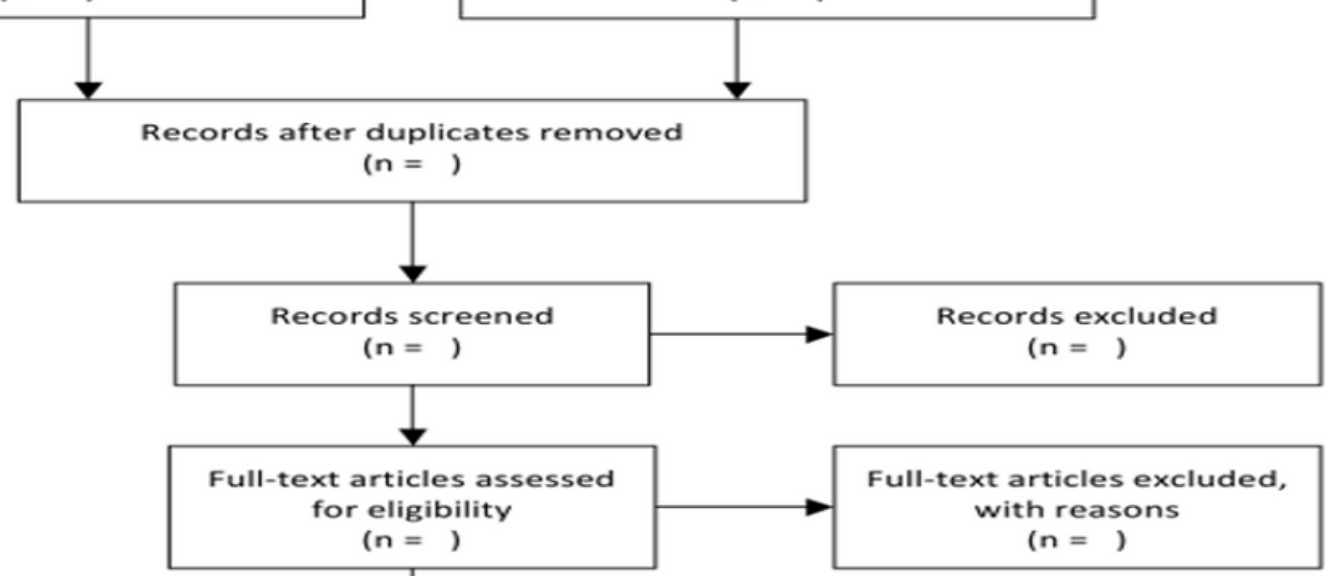

Studies included in qualitative synthesis $(n=)$

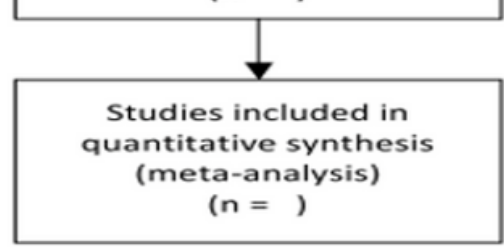

Figure 1

PRISMA flow diagram 


\section{Supplementary Files}

This is a list of supplementary files associated with this preprint. Click to download.

- Appendices.docx 